\title{
LOCALIDADES DE LA PICEA CHIHUAHUANA
}

\section{Por MAXIMINO MARTINEZ}

de la S. B. M.

En 1942, en que fue descubierta en México la Picea chihuahuana, solamente se registraron cuatro lugares del estado de Chihuahua (Anales del Instituto de Biología XIII. 1. 31. 1942).

Desde aquel año a esta fecha se ha encontrado en otras localidades, y aquí se mencionan todas:

Arroyo de Los Talayotes, Predio Tuteáchic, Municipio de Bocoyna, Chih.

a 2350 metros. (Rigoberto Dueñas. 3 de febrero de 1942).

Arroyos de Urichique, El Cuervo y Meguáchic, Municipio de Bocoyna,

Chih. (Rigoberto Dueñas. 9 de octubre de 1942).

Martínez M. 1957. Localidades de la Picea chihuahuana. Boletín de la Sociedad Botánica de México 21: 33-34. 
Cituriáchic, a $8 \mathrm{Km}$. de San Juanito, Chih., a 2450 metros. (Rigoberta Dueñas. 10 de mayo de 1943).

Margen izquierda del río Temochic, Municipio de Guerrero, Chih. (Rigoberto Dueñas. 30 de mayo de 1943).

Cerro de La Cruz, a 500 metros de San Juanito, Chih. a 2400 metros. (Rigoberto Dueñas. 12 de junio de 1942).

Reserva Nacional de Tutuaca, Municipio de Temosáchic, Chih. (Emilio Flores Calderón. 15 de octubre de 1948).

El Vergel, Municipio de Balleza, casi en el límite de los Estados de Chihuahua y Durango, a 2500 metros. (J. J. Villa Jr. 21 de febrero de 1949).

Arroyo de Santa Bárbara, Pueblo Nuevo, Dgo. (Francisco Lozoya. 14 de octubre de 1948).

Arroyos de Santa Bárbara y Hornitos, Bosques de El Salto, Pueblo Nuevo, Dgo. (Cenobio E. Blanco. 20 de septiembre de 1947).

Sierra de La Candela, Tepehuanes, Dgo. (Francisco Lozoya. 2 de octubre de 1947).

Arroyo de La Laguna, Bajíos de D. Víctor, Municipio de Durango, Dga. (Julián Fernández. 12 de abril de 1957). 